\title{
An Instruction on the In Vivo Shell-Less Chorioallantoic Membrane 3-Dimensional Tumor Spheroid Model
}

\author{
Nzola De Magalhães · Lih-Huei L. Liaw • \\ Michael Berns
}

Received: 20 September 2009/ Accepted: 7 May 2010/Published online: 25 May 2010

(C) The Author(s) 2010. This article is published with open access at Springerlink.com

\begin{abstract}
The traditional shell chicken chorioallantoic membrane (CAM) model has been used extensively in cancer research to study tumor growth and angiogenesis. Here we present a combined in vivo tumor spheroid and shell-less CAM three-dimensional model for use in quantitative and qualitative analysis. With this model, the angiogenic and tumorigenic environments can be generated locally without exogenous growth factors. This physiological model offers a stable, static and flat environment that features a large working area and wider field of view useful for imaging and biomedical engineering applications. The short experimental time frame allows for rapid data acquisition, screening and validation of biomedical devices. The method and application of this shell-less model are discussed in detail, providing a useful tool for biomedical engineering research.
\end{abstract}

Electronic supplementary material The online version of this article (doi:10.1007/s10616-010-9279-8) contains supplementary material, which is available to authorized users.

N. De Magalhães (凶)

Biomedical and Chemical Engineering, Rochester Institute of Technology, Rochester, NY, USA

e-mail: ndmbme@rit.edu

L.-H. L. Liaw · M. Berns

Beckman Laser Institute and Medical Clinic, University of California Irvine, Irvine, CA, USA
Keywords Chicken chorioallantoic membrane · CAM $\cdot$ Spheroid $\cdot$ Shell-less $\cdot$ Biomedical $\cdot$

Tumor

\section{Introduction}

Effective investigations in cancer research, whether in tumor dynamics, drug delivery, drug or diagnostic tool development involves the use biological models that reflect physiologic characteristics of solid tumors. Three-dimensional in vitro models and in vivo models are increasingly used to study tumor growth and for diagnostic and therapeutic screening applications (Kanzawa and Saijo 1997; Rupniak et al. 1983; Zietarska et al. 2007). Three-dimensional in vitro models include cultured biopsies, and multilayer cultures such as cell clusters and spheroids (Sonoda et al. 2003). While there are currently no high throughput in vivo models, the use of the chick CAM has become popular as 'moderate throughput' screening procedure (Ribatti et al. 2001). However, a major limitation of this in vivo model is time constraint, and thus tumor growth is limited in size and relative maturation.

Three-dimensional spheroids are tissue engineered from a small cluster of individual cells that in suspension in culture medium grow into natural multilayered spheroid bundles (Santini and Rainaldi 1999). Unlike cell monolayers, in vivo solid tumors grow in a three dimensional fashion, with different 
areas of the tumor subject to different nutrient availability and environmental signals. Because oxygen can diffuse efficiently into the tissue up to $200 \mu \mathrm{m}$ in distance, cell regions more than $200 \mu \mathrm{m}$ away from oxygen and nutrient supply frequently become necrotic, and solid tumors are often found to contain necrotic regions due to nutrient deficiency (Santini and Rainaldi 1999). Tumor spheroids mimic the early growth and morphological dynamics of solid tumors. When tumor spheroids grow over approximately $200 \mu \mathrm{m}$ in radius, nutrients cannot diffuse readily to the center of the spheroids. This creates a unique cellular spatial conformation across the spheroid; the outer layer is populated with proliferating cells due to their direct contact with surrounding nutrients and oxygen; the center develops a necrotic core like solid tumors due to hypoxia created by low oxygen content; and the layer between center and the outer layer is populated by quiescent or non-proliferating cells. Though avascular, tumor spheroids can mature to sizes ranging from 1 to $2 \mathrm{~mm}$ in diameter. This is typically the size at which an angiogenic switch would be manifested in a successful, aggressive tumor. It is beyond this stage that further growth of spheroids in vitro is of diminishing value.

Here, we have exploited the fact that spheroid tumors can develop for several weeks in an avascular microenvironment (in vitro). Transplantation of these preformed tumors into the CAM model extends the utility of the CAM, permitting the study of later stages in tumor development than otherwise possible. The symmetric 3D configuration of tumor spheroids facilitates the generation of boundary conditions and fixed data points useful in quantitative investigations, such as computational modeling of tumor growth (Chaplain 1996; Liang et al. 2005). Moreover, the ability to use spheroids rather than biopsy fragments for this purpose allows for genetic manipulation of the tumors cells not easily accomplished with tumor biopsies.

The traditional CAM model, first introduced as the shell model (Knighton et al. 1977), remains useful for various tumor biology studies. However, it is not optimal for imaging and biomedical engineering investigations due to restricted access to tumor sites and the relative unstable environment of shell (both due to fragility and the ability of the embryo to move freely in the presence of perturbations). Drug injections and imaging studies can be challenging. Here, we describe the combined use of spheroids in the shell-less assay (Jakobson et al. 1989; Ono 2000), which has the advantages of stability, ease of access, large area and amenability to serial quantitative analysis (Online Resource 1). The model provided is robust and versatile, allowing adaptation for studies of tumor pathology and therapeutic efficacy.

\section{Materials and methods}

\section{3-D spheroid preparation}

To create in vitro cultures of spheroids, we use a variation of the liquid-overlay technique (Santini and Rainaldi 1999). Single cell suspensions $(0.5 \times$ $10^{6}$ tumor cells $/ \mathrm{ml}$ ) are added to non-treated agar coated (2\%) square Petri dishes (BD Falcon square Petri dish) $100 \times 15 \mathrm{~mm}$ filled with culture medium, and incubated at $37{ }^{\circ} \mathrm{C}$ and $7.5 \% \mathrm{CO}_{2}$. After 3 days of incubation, tumor cell aggregates are transferred to non-agar coated (regular) Petri dishes filled with cell culture medium, and grown to tumor spheroids of approximately $500 \mu \mathrm{m}$ to $1.0 \mathrm{~mm}$ in diameter (Online Resource 2).

\section{Shell-less CAM preparation}

Shell-less CAM preparation is conducted on day 3 of embryonic development (EA). Fertilized white (Leghorn, lab grade) or brown (Rhode Island, lab grade) chicken eggs (AA Lab Eggs, Inc, Westminster, CA), disinfected with $70 \%$ alcohol or germicidal cloths (Sani-Cloth Plus, PDI: The Healthcare Division of Nice-Pak Products, Inc., Orangeburg, NY), are incubated in a static ventilated hatching incubator set at $38{ }^{\circ} \mathrm{C}$ and $60 \%$ humidity.

Under clean and light restricted conditions, the contents of the egg are carefully dropped by force of gravity in a dry and sterile condiment dish $4.5 \mathrm{~cm}$ diameter (bottom), $6.5 \mathrm{~cm}$ diameter (top), $4.5 \mathrm{~cm}$ height. Dishes are available from several viable suppliers including Wal-Mart.

The dish is subsequently covered with a breathable polyethylene sheet (Fisher brand all-purpose laboratory wrap polyvinyl-chloride, Fisher Scientific, Pittsburgh, PA), and incubated. Additional holes can be added using an $18 \mathrm{G}$ needle to ensure gas exchange. 
The dish is incubated until days 7 or 8 EA for spheroid implantation. The detailed protocol of the technique can be found under the supplemental materials (Online Resource 3).

Tumor spheroid implantation

Tumor spheroid implantation is conducted on day 7 or 8 of embryonic development. Under sterile conditions, a small incision is made using a 30 gauge hypodermic needle attached to a $1 \mathrm{cc}$ syringe, in the upper surface (ectodermal epithelium) of the CAM in a region away from major blood vessels. A small area of the chorionic layer of the CAM is scratched with the edge of the needle's bevel. The ectodermal epithelium has been successfully removed when the target area appears watery due to local ischemia. Any resulting sera, blood or debris is gently aspirated with the syringe. Finally, one tumor spheroid of $500 \mu \mathrm{m}^{3}$ to $1.0 \mathrm{~mm}^{3}$ in size is dropped onto the severed area using a spatula (Online Resource 4). The dish is then covered with a semi-porous sheet, and returned to the incubator.

Successful implantation is observed when the tumor spheroid is engulfed by the CAM (typically 2 days post-implantation), and the tumor spheroid rests in the highly vascularized mesodermal layer between the allantoic epithelium (endoderm) and the healed chorionic epithelium (ectoderm). At this stage, the model is ready for use in biological and biomedical engineering investigations.

\section{Discussion}

Vascularized tumor spheroids from a variety of human derived tumor cell lines have been generated using this method including MCF-7 breast cancer, BXPC-3 pancreatic cancer, NB5 neuroblastoma, U-87 and ACBT (ACBT-G., Granger, University of California, Irvine) glioblastoma cell lines.

Microvasculature in the center region of the surface of the spheroid during day 14 of embryonic stage is evident in Fig. 1a. Five distinct microvessels were found at the center of the spheroid (Fig. 1b) upon examination of the middle histological section of the H\&E stained CAM/tumor spheroid tissue. Invasion of the tumor cells to adjacent areas of the CAM is also observed.

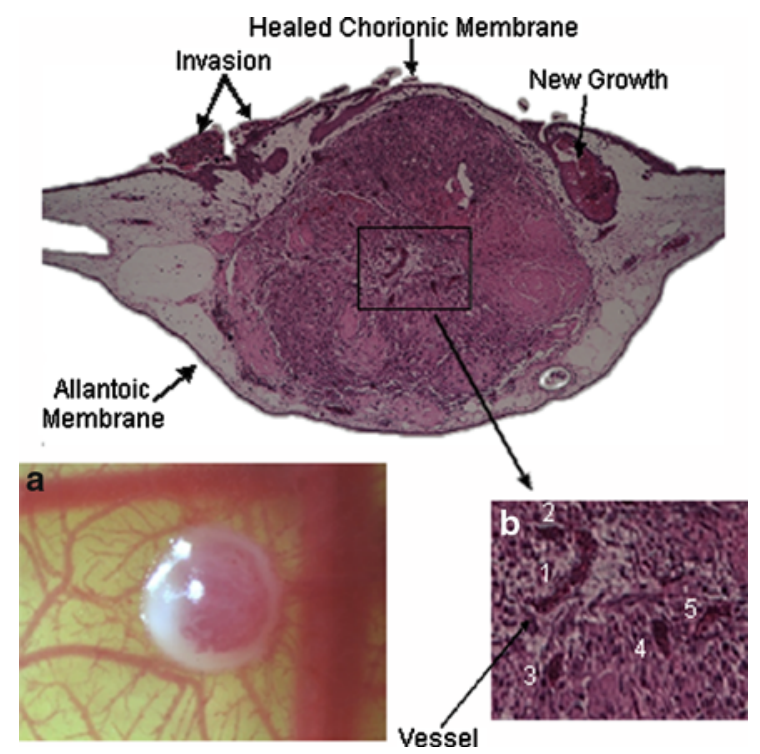

Fig. 1 a Top view of tumor spheroid and CAM interface-a $1 \mathrm{~mm}$ diameter ACBT glioblastoma spheroid inside CAM (mesoderm) on live chicken embryo on day 14 EA; b H\&E histological section of $1 \mathrm{~mm}$ diameter glioblastoma spheroid embedded in CAM. Grey area is the CAM; center region in purple denotes the tumor spheroid. Other purple regions denote tumor cells. Tumor microvasculature as a result of angiogenesis (1-5), and new tumor invaded regions are observed

Figure 1a shows successful development of the tumor vascular network using this CAM/tumor model without the use of exogenous growth factors. Although there is presence of angiogenic activity on the CAM due to embryogenesis and wound healing of the chorionic epithelium, penetration of the blood vessels to the center of the tumor is evidence that vessel growth into the tumor is induced by angiogenic factors endogenous to the tumor. High angiogenic activity was observed in spheroids of $500 \mu \mathrm{m}-1 \mathrm{~mm}$ in diameter as opposed to $2 \mathrm{~mm}$ spheroids (not shown). It is possible that spheroids larger than $1 \mathrm{~mm}$ diameter are inefficiently embedded in the CAM. Therefore, the top portion of the spheroid would not be nourished by the mesoderm fluid and would dry out. Spheroids smaller than $500 \mu \mathrm{m}$ are more challenging to spot with the naked eye due to their size and transparency as they are less compact than larger spheroids. Thus, they can be difficult to locate on the CAM post implantation. Spheroids between $500 \mu \mathrm{m}$ and $1 \mathrm{~mm}$ tend to yield optimal results.

Figure 1 shows the presence of new tumors, evidence of breakdown and clearance of the necrotic 
core, and invasion of the tumor cells in surrounding areas of the CAM. These results suggest that this CAM/tumor model can also be used to study tumor invasion and metastasis in addition to tumor growth and angiogenesis.

The efficacy of drugs can also be tested using this CAM/tumor model (Vargas et al. 2007). In therapeutic and imaging studies, depending on the goal of the investigation, drugs are often administered to the system topically, intraperitoneally or intravenously. Nanoparticles carrying therapeutic drugs can be introduced into the CAM vascular system via the intraperitoneal and intravenous delivery techniques. With our spheroid based shell-less CAM model, drug and therapeutic efficacy as well as the potential of nanoparticle target selectivity and delivery for cancer treatment can be assessed. In the topical delivery method, the drug is administered directly onto the target site without invasive perturbation the CAM. Alternatively, slow release gels (depot effect) containing a drug of choice can be applied on the CAM/ spheroid interface. While the topical technique provides fast and direct delivery of the drug to the target site, it does not mimic drug applications for cancer treatment in a clinical setting. Intraperitoneal and intravenous delivery methods are invasive techniques that most closely match the clinical setting. Unlike the topical application where the actual concentration of the drug delivered reaches the target, the intraperitoneal application results in slower delivery as the drug is first absorbed by the host and subsequently the dosage may be lower when it reaches the target tumor. In addition, intraperitoneal delivery can result in lower survival rate because critical organs of the embryo may be compromised. Intravenous delivery provides rapid drug delivery into the closed vascular network of the CAM.

A drawback of the shell-less CAM model is that survival rate of the embryo is somewhat lower than of the shell CAM due in part to the absence of calcium supply during the embryo development (Dunn et al. 1987). To overcome this drawback, the number of embryos used during the experiments should be adjusted to match the data requirements of the individual research. In addition, the relatively short experimental window (approximately 10 days after implantation) over which experiments can be conducted due to the fixed embryonic stage of the chicken embryo, limits the model to short term studies. The shell-less tumor spheroid based CAM model should therefore be used as a transitional model to obtain rapid validation of biomedical engineering applications, and studies of long term effects of the applications should be conducted in alternate in vivo models feasible for these types of studies.

By combining two classical systems, the tumor spheroid and the shell-less CAM, we have developed an in vivo model that is not only easily adaptable and applicable to many scientific fields, but one which can provide fast and efficient means to quantitatively investigate cancer pathology, test developing noninvasive medical imaging technologies, and evaluate new therapies.

Acknowledgments Financial support for this project was provided by the NIH F31 Grants CA12371-01 and CA1237102, and the Merck-UNCF predoctoral fellowship. This article was made possible by the tremendous generosity, expertise and mentorship of the honorable Mrs. Li-Huei Liaw. The authors thank Linda $\mathrm{Li}$ and Angela Giogys for the valuable contributions during the data processing stage. We also extend our gratitude to Dr.Tromberg and Dr. Berns for the use of the Beckman Laser Institute facility, Dr. Stupack and Dr. Lowengrub for assistance with manuscript editing and structure.

Open Access This article is distributed under the terms of the Creative Commons Attribution Noncommercial License which permits any noncommercial use, distribution, and reproduction in any medium, provided the original author(s) and source are credited.

\section{References}

Chaplain MAJ (1996) A vascular growth, angiogenesis and vascular growth in solid tumors: the mathematical modeling of the stages of tumor development. Math Comput Modeling 23:47-87

Dunn BE, Clark NB, Scharf KE (1987) Effect of calcium supplementation on growth of shell-less cultured chick embryos. J Exp Zool Suppl 1:33-37

Jakobson AM, Hahnenberger R, Magnusson A (1989) A simple method for shell-less cultivation of chick embryos. Pharmacol Toxicol 64:193-195

Kanzawa F, Saijo N (1997) In vitro interaction between gemcitabine and other anticancer drugs using a novel threedimensional model. Semin Oncol 24:8-16

Knighton D et al (1977) Avascular and vascular phases of tumor growth in the chick embryo. Br J Cancer 35:347356

Liang Y, Pjesivac-Grbovic J, Cantrell C, Freyer JP (2005) A multiscale model for avascular tumor growth. Biophys $\mathrm{J}$ 89:3884-3894 
Ono T (2000) Exo ovo culture of avian embryos. Methods Mol Biol 135:39-46

Ribatti D, Nico B, Vacca A, Roncali L, Burri P, Djonov V (2001) Chorioallantoic membrane capillary bed: a useful target for studying angiogenesis and anti-angiogenesis in vivo. Anat Rec 264:317-324

Rupniak HT, Dennis LY, Hill BT (1983) An intercomparison of in vitro assays for assessing cytotoxicity after a 24 hour exposure to anti-cancer drugs. Tumori 69:37-42

Santini M, Rainaldi G (1999) Three-dimensional spheroid model in tumor biology. Pathobiology 67:148-157
Sonoda $\mathrm{T}$ et al (2003) Expression of angiogenesis factors in monolayer culture, multicellular spheroid and in vivo transplanted tumor by human ovarian cancer cell lines. Cancer Lett 196:229-237

Vargas A et al (2007) The chick embryo and its chorioallantoic membrane (CAM) for the in vivo evaluation of drug delivery systems. Adv Drug Deliv Rev 59:1162-1176

Zietarska M et al (2007) Molecular description of a 3D in vitro model for the study of epithelial ovarian cancer (EOC). Mol Carcinog 46:872-885 